\title{
Articles
}

\section{Non-Motor Symptoms of Essential Tremor Are Independent of Tremor Severity and Have an Impact on Quality of Life}

\author{
Thomas Musacchio $^{1}{ }^{1}$, Veronika Purrer ${ }^{1}$, Aikaterini Papagianni ${ }^{1}$, Anna Fleischer ${ }^{1,2}$, Daniel Mackenrodt ${ }^{1}$, Carolin Malsch $^{2}$, Götz Gelbrich $^{2,3}$, \\ Frank Steigerwald ${ }^{1}$, Jens Volkmann ${ }^{1}$ \& Stephan Klebe ${ }^{1,4 *}$ \\ ${ }^{1}$ Department of Neurology, University Hospital of Würzburg, Würzburg, Germany, ${ }^{2}$ Institute of Clinical Epidemiology and Biometry, University of Würzburg, \\ Würzburg, Germany, ${ }^{3}$ Clinical Trial Center, University Hospital of Würzburg, Würzburg, Germany, ${ }^{4}$ Department of Neurology, University Hospital of Freiburg, \\ Freiburg, Germany
}

\begin{abstract}
Background: Several publications have focused on accompanying non-motor symptoms (NMS) in essential tremor (ET) patients; however, it remains unclear if NMS are an intrinsic part of the disease or secondary phenomena. We present the results of several neuropsychiatric tests and their impact on quality of life (QoL) in community-dwelling patients with ET.

Methods: Participants were recruited via a newspaper article about ET published in the local media and on the internet. All participants completed several standard neuropsychiatric tests, including those that assess QoL. To compare differences between cases and controls, Student's $t$-tests with Bonferroni-Holm post hoc tests were performed. Spearman's correlation coefficients were also calculated.

Results: We enrolled 110 patients with definite or probable ET. Highly significant changes were observed for apathy, anxiety, and cognition and negatively impacted QoL. Most aberrations were independent of tremor severity and duration.

Discussion: The significant neuropsychiatric deficits and reduced QoL demonstrate a degree of illness that appears to be a non-motor phenotype rather than a secondary effect of ET. In the future, NMS should carefully be explored in ET patients as they may have an impact on QoL and treatment.
\end{abstract}

Keywords: Essential tremor, non-motor symptoms, quality of life

Citation: Musacchio T, Purrer V, Papagianni A, etal. Non-motor symptoms of essential tremor are independent of tremor severity and have an impact on quality of life. Tremor Other Hyperkinet Mov. 2016; 6. doi:10.7916/D8542NCH

*To whom correspondence should be addressed. E-mail: stephan.klebe@uniklinik-freiburg.de

"These authors contributed equally to this work.

Editor: Elan D. Louis, Yale University, USA

Received: November 23, 2015 Accepted: January 19, 2016 Published: March 8, 2016

Copyright: ๑ 2016 Musacchio et al. This is an open-access article distributed under the terms of the Creative Commons Attribution-Noncommercial-No Derivatives License, which permits the user to copy, distribute, and transmit the work provided that the original authors and source are credited; that no commercial use is made of the work; and that the work is not altered or transformed.

Funding: None.

Financial Disclosures: There was no funding/sponsorship for this study. T.M. received funding for a trip from AbbVie and Medtronic. V.P., A.P., A.F., D.M., C.M., and G.G. report no disclosures. F.S. received research support from UCB, Medtronic, Boston Scientific, and SIMI-Motion and royalties from Archimedes Pharma and Boston Scientific. J.V. receives research support from Medtronic and Boston Scientific and royalties from Medtronic, Boston Scientific, St. Jude, TEVA, UCB, AbbVIE, and Allergan. S.K. received travel funding from $\mathrm{UCB}$ and AbbVie and royalties from Actelion, UCB, and Thieme.

Conflict of Interest: The authors report no conflict of interest.

Ethics Statement: This study was performed in accordance with the ethical standards detailed in the Declaration of Helsinki. The authors' institutional ethics committee has approved this study and all patients have provided written informed consent.

\section{Introduction}

Essential tremor (ET) is one of the most common movement disorders with a well-known and clearly defined motor phenotype. ${ }^{1}$ Firstly described and understood as a monosymptomatic disease, ${ }^{2}$ our understanding of ET symptomatology has shifted to a more complex and multidimensional phenotype. ${ }^{3}$ ET pathology and the involved central nervous regions are uncertain. There is some evidence that ET could be a neurodegenerative disease ${ }^{4}$ with pathologic abnormalities reported in the cerebellum ${ }^{5}$ and brain stem, ${ }^{6}$ but the results are inconsistent, especially regarding Purkinje cell loss. Conversely, one hypothesis is that ET is a functional disorder of subcortical circuits without neurodegeneration. ${ }^{7}$ One factor common to these pathophy- 
siologic concepts is that they are not restricted to motor symptoms; there can be additional symptoms besides the motor phenotype. In the last few years, several publications have focused on accompanying non-motor symptoms (NMS) with a variety of cognitive and neuropsychiatric changes in ET patients. The reported abnormalities include mild cognitive impairment, ${ }^{8-10}$ apathy, ${ }^{11}$ depression, ${ }^{12,13}$ anxiety, ${ }^{14,15}$ sleep disturbances, ${ }^{16}$ and reduced quality of life (QoL). ${ }^{17}$ It remains unclear if NMS are an intrinsic part of the condition, as in Parkinson's disease (PD) or Huntington's disease, or if they are secondary phenomena due to disease duration or severity. Most published studies have focused on only one specific or a few NMS. Here we attempted to define the pattern of NMS in ET and present the results of a large variety of neuropsychiatric tests and their impact on QoL as a test battery in ET patients recruited from the community.

\section{Methods}

\section{Patient enrollment}

Participants were recruited in a first step by an informative newspaper article about ET published in the local media ("Mainpost," 157,000 copies) and on the website of University Hospital Würzburg, Germany. The article was combined with an appeal to all readers who subjectively suffered from tremor to take part in a neuropsychiatric study. After an initial contact by phone, all interested individuals were invited to a screening and inclusion visit. In preparation for the screening visit, the participants received a selfquestionnaire by mail. In total, 214 subjects completed the screening. The study was approved by the Ethics Committee of the Würzburg Medical Faculty and the Ethical Board of the Physician's Association Hessen and was conducted in accordance with the declaration of Helsinki. All participants provided written, informed consent.

\section{Neurological examination and tremor diagnosis}

At the screening visit, all participants underwent medical history taking and clinical examination by a movement disorder specialist (T.M., A.P., or S.K.). ET was diagnosed as probable, possible, or definite as defined by the consensus criteria of the Tremor Investigation Group (TRIG). ${ }^{18}$ Tremor was quantified using the Fahn-Tolosa-Marin Tremor Rating Scale (FTRS). To ensure the quality of the data, the intra- and inter-rater reliabilities for Archimedes spirals using intraclass coefficient were calculated.

\section{Neuropsychiatric assessment}

QoL with the Short-Form 36-Item Health Survey (SF-36). The ShortForm 36-Item Health Survey (SF-36) was used to assess QoL. This is a very common tool used to measure mental health and was validated in a normal German population in a large survey. ${ }^{19,20}$ The SF-36 consists of eight subdomains divided and grouped into a mental domain (vitality, social function, role emotional, and mental health) and a physical domain (physical function, role physical, bodily pain, and general health). Each subdomain, as well a physical component score (PCS) and mental component score (MCS), can be scored, with higher scores indicating higher QoL.
Apathy Evaluation Scale (AES). To assess the clinical symptom of apathy, defined as a lack of motivation and diminution of goal-directed behavior, we used the validated and widely-used Apathy Evaluation Scale (AES), which is an 18-item self-questionnaire that measures apathy over the past 4 weeks. $^{21}$ The total score ranges from 18 to 72 points, with higher values indicating more severe apathy. The mean score of a healthy control group is 23.5 points $(\mathrm{SD}=5.8, n=37$, mean age 65.2 years). ${ }^{22}$

State-Trait Anxiety Scale (STAI-T). The State-Trait Anxiety Scale (STAI-T) was used to measure the symptom anxiety as a characteristic trait. We assessed "trait" anxiety by using items 21-40 of the original test. Each item of the self-questionnaire can be rated on a 4-point intensity scale. The mean values are adjusted for age and sex, and higher values indicate more trait anxiety. ${ }^{23}$

Beck Depression Inventory (BDI). The Beck Depression Inventory (BDI) is commonly used to measure depressive symptoms in the last week and has been revised several times in the last few decades. The current version consists of 21 items; the patient scores each from 0 to 3 , and the results are summed. Higher total scores indicate more severe depressive symptoms. Validated cut-offs separate normal values from severe depressive symptoms. ${ }^{24}$

Epworth Sleepiness Scale (ESS). To assess subjective daytime sleepiness, we used the Epworth Sleepiness Scale (ESS) selfquestionnaire consisting of a depiction of eight situations that are scored from 0 to 3 . The sum scores have been validated in a German cohort, with a normal value for healthy controls of 5.7 points $(\mathrm{SD}=$ $3.0, n=159$, mean age 35 years), and higher scores in patients with various sleep disorders reflecting subjective sleep propensity. ${ }^{25}$

Frontal Assessment Battery (FAB). The Frontal Assessment Battery $(\mathrm{FAB})$ is a practicable and validated instrument to test executive dysfunction and consists of six subtests (conceptualization, mental flexibility, motor programming, sensitivity to interference, inhibitory control, and environmental autonomy). For healthy controls, a mean value of 17.3 points ( $\mathrm{SD}=0.8, n=42$ ) was reported in an Englishspeaking control group, and lower scores indicate executive dysfunction. ${ }^{26}$ A trained rater (V.P. or S.K.) administered the FAB.

\section{Parkinson Neuropsychometric Dementia Assessment (PANDA)} (cognitive subscore). The Parkinson Neuropsychometric Dementia Assessment (PANDA) is a broadly used and validated instrument to detect cognitive deficits; it was specially designed for subjects with PD. ${ }^{27}$ It consists of five tasks including verbal fluency, word pair association learning with immediate and delayed recall, visuospatial perception, and working memory testing. There are validated cut-offs, with scores lower than 18 points suspicious for mild cognitive impairment and a mean score of 23.6 points $(\mathrm{SD}=5.4, n=108$, mean age 60.3 years) for healthy subjects. ${ }^{27} \mathrm{~A}$ trained rater (V.P. or S.K.) administered the PANDA. 
Olfactory testing with Sniffin Sticks. Standardized Sniffin Sticks that are commonly used in PD and other non-neurological disorders were used to test olfactory function. The test is based on pen-like, odordispensing devices, and we used the "Screening 12 Test" for odor identification. The resulting values were compared to age-matched values from a healthy control group to identify subjects with olfactory dysfunction. ${ }^{28}$

\section{Statistical analysis}

In order to examine ET-induced QoL decreases, our findings were compared to reference data from the German general population (hereinafter referred to as "healthy controls") as provided in the manuals of the questionnaires. The $p$-values and 95\% confidence intervals (CIs) for mean differences were computed using the Student's $t$-statistic. For SF-36 domains, STAI-T and Sniffin Sticks, we employed age- and sex-specific German reference data; $p<0.05$ was considered statistically significant. To account for multiple tests, differences that remained significant after Bonferroni-Holm adjustment are indicated. Relationships between age, tremor characteristics, and NMS scores were assessed using Spearman's correlation coefficient $r$. All statistics were performed in SPSS (version 22).

Table 1. Glinical-demographic Data of 110 ET Cases ${ }^{1}$

\begin{tabular}{|lc}
\hline Characteristics & Cases \\
\hline Age, y & $65.2 \pm 14.1$ \\
Sex, M & $65(59.1 \%)$ \\
Age of onset, y & $41.4 \pm 21.0$ \\
Disease duration, y & $23.8 \pm 16.4$ \\
FTRS score & $36.8 \pm 14.6$ \\
Familial ET & $82(74.5 \%)$ \\
Alcohol sensitivity & \\
Responder & $49(44.5 \%$ \\
Non-responder & $40(36.4 \%)$ \\
Unknown & $21(19.1 \%)$ \\
\hline
\end{tabular}

Topographical distribution of tremor

\begin{tabular}{|lc}
\hline Upper extremities & $110(100 \%)$ \\
Voice & $51(46.4 \%)$ \\
Head & $46(41.8 \%)$ \\
Lower extremities & $18(16.4 \%)$ \\
& \\
& ${ }^{1}$ Values are presented as mean \pm SD or number (\%). \\
Abbreviations: ET, Essential Tremor; FTRS, Fahn-Tolosa- \\
Marin Tremor Rating Scale; M, Male; y, Years.
\end{tabular}

\section{Results}

\section{Epidemiology and clinical characteristics}

Detailed patient characteristics are shown in Table 1. From the 214 patients at the screening visit, 110 patients (65 males) with definite or probable ET were enrolled and completed the entire test battery (103 patients with definite ET; 7 patients with probable ET) (Figure 1). We excluded 104 patients because they either did not fulfill the criteria for probable or definite ET or suffered from other tremor conditions (i.e., parkinsonian, dystonic, psychogenic) (Figure 1). The intraclass coefficients for intra- and inter-rater reliabilities for Archimedes spirals ranged from 0.93-0.95. Regarding the topographical distribution of tremor, all $110(100 \%)$ patients displayed typical symmetric action tremor of the upper extremities, $51(46.4 \%)$ had an associated voice tremor, $46(41.8 \%)$ exhibited a head tremor, and 18 (16.4\%) had an action tremor of the lower extremities.

\section{Neuropsychiatric and QoL scales}

The results of the neuropsychiatric and QoL scales are summarized in Table 2. The SF-36 results are graphically depicted in Figure 2, and the AES, STAI-T, FAB, and PANDA scores are shown in Figure 3.

QoL measured by SF-36. Our group of ET patients showed no significant differences in the PCS with 2.3 (CI 0.4, 4.2; $p=0.017$ ) after Bonferroni-Holm correction, but they did show a highly significant reduction of -6.4 (CI $-8.5,-4.3 ; p<0.001)$ points in the MCS compared with healthy, age-matched German controls. The subanalysis of the significant MCS domains showed maximum differences in "role emotional" $(p<0.001)$ followed by "social function" ( $p<$ $0.001)$ and "mental health" $(p<0.001)$. "Vitality" $(p=0.006)$ showed no significant change. Only the subdomains "social function" $(r=$ $-0.27, p=0.005)$ and "mental health" $(r=-0.22, p=0.025)$ correlated with the total FTRS score. "Role emotional" showed no correlation with age, sex, age of onset (AOO), disease duration (DD), or total FTRS score. Overall, MCS did not significantly correlate to any attribute of the study population. For overall PCS, we found a negative correlation with the total FTRS score.

AES. Compared to the normal control group, the mean total score in AES was higher by 9.5 (CI 6.6, 12.5) points, with a mean score of 33.0. This result was highly significant $(p<0.001)$, indicating more apathy in ET patients. There was no correlation with age, sex, AOO, DD, or total FTRS score.

STAI-T. The STAI-T in our study population was 39.8 and was significantly higher by 3.7 points (CI 1.6, $5.9 ; p=0.001$ ) compared to a normal population adjusted for age and sex. This suggests more trait anxiety in ET patients. There was no correlation with age, sex, AOO, DD, or total FTRS score.

BDI. The BDI scores were 8.0 points in ET patients, with a difference of 1.5 points (CI $-0.2,3.2$ ) compared to a normal population. However, this was not statistically significant $(p=0.081)$. 


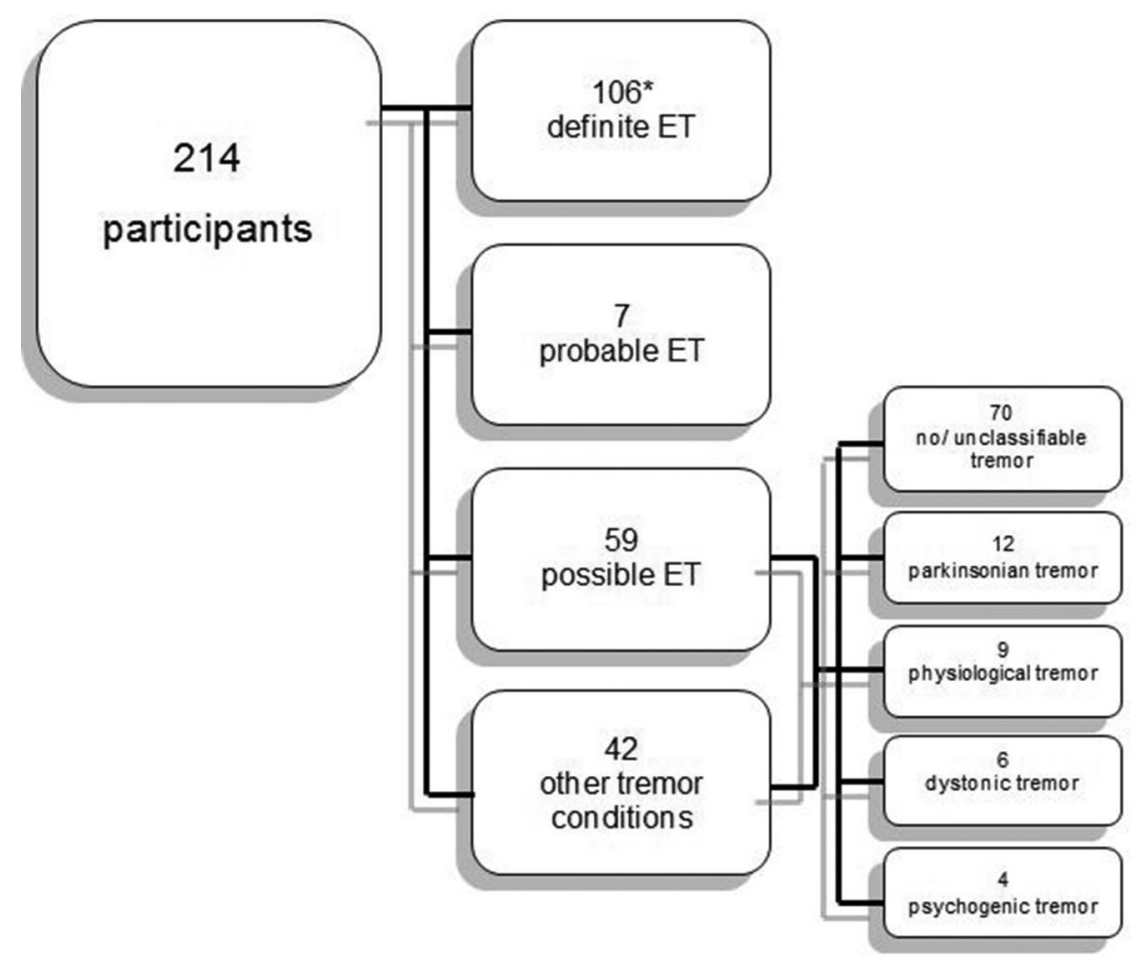

* 3 participants were unable to perform all neuropsychatric test batteries correctly and were excluded from further analysis.

Figure 1. Flowchart Depicting the Diagnosis of All Recruited Participants

ESS. Regarding daytime sleepiness, we found a non-significant difference of 0.9 points (CI 0.1, 1.7; $p=0.021$ ) after BonferroniHolm correction. The total mean score in our population was 6.6.

FAB. Results of frontal cognitive dysfunction testing showed a highly significant difference of -1.1 points (CI $-1.5,-0.7 ; p<0.001$ ), with a mean score of 16.2 points in our study population. This was in the normal range of healthy controls. There was a positive correlation with age $(r=-0,41, p<0.001)$ and AOO $(r=-0.25, p=0.009)$, but no correlation with sex, DD, or total FTRS score.

PANDA-cognition. Compared to a normal population, the mean score in our patients was 19.3 points, which corresponded to a highly significant $(p<0.001)$ difference of -4.3 points (CI $-5.8,-2.9$ ) indicating a tendency to mild cognitive impairment. Correlations were found regarding age $(r=-0.30, p=0.002)$, AOO $(r=-0.21 p=$ 0.029), and total FTRS score $(r=-0.39, p<0.001)$.

Sniffin Sticks. Adjusted to age, there was no statistically significant difference between the results in ET patients and the normal population data. We observed a small absolute reduction of -0.3 points $(p=0.139)$.

Test battery correlation analysis. Analyzing the Spearman correlations among the tests themselves indicated that similar measures were highly correlated; thus, this can be used as a quality indicator for the reliability of our assessment technique. The highest correlation coefficients were found between MCS/STAI-T $(r=0.78, p<0.001)$, BDI/STAI-T $(r=0.70, p<0.001)$, AES $/$ STAI-T $(r=0.62, p<0.001)$, and AES/BDI $(r=0.60, p<0.001)$. AES itself correlated with ESS $(r=0.33, p=0.001)$ and PANDA $(r=-0.34, p<0.001)$, and PANDA-cognition and FAB were also significantly correlated $(r=0.42, p<0.001)$. All of these correlations are listed in Table 3.

\section{Discussion}

Reports in the few last years have described NMS in patients suffering from ET. There are additional features accompanying the disease besides pure motor abnormalities. ${ }^{29}$ Most investigations published to date have focused on isolated symptoms including neuropsychiatric abnormalities (e.g., cognitive impairment ${ }^{30}$ and depression ${ }^{13,14}$ ) and QoL. ${ }^{17}$ Another interesting and persistent question is whether NMS in ET are a primary disease feature or secondary reactive symptoms. ${ }^{31}$ Most investigations have been unable to answer this question because either the study population was relatively small ${ }^{12,32}$ or the NMS correlated with tremor severity or duration. ${ }^{13}$ Another important limitation in most previous studies was subject recruitment, which mainly occurred among outpatients in specialized movement disorder units, and a consecutive selection 
Table 2. Non-motor Parameters among Patients with ET and Controls

\begin{tabular}{|c|c|c|c|c|c|c|c|c|c|c|}
\hline \multirow{3}{*}{ SF-36 domain } & \multicolumn{3}{|c|}{ ET Cases } & \multicolumn{3}{|c|}{ Control Subjects ${ }^{1}$} & \multirow{3}{*}{$\begin{array}{c}\text { Mean } \\
\text { Difference }\end{array}$} & \multirow{2}{*}{\multicolumn{2}{|c|}{$\begin{array}{l}\text { 95\% Confidence } \\
\text { Interval }\end{array}$}} & \multirow[t]{3}{*}{$\boldsymbol{P}^{7}$} \\
\hline & \multirow[t]{2}{*}{$n$} & \multirow[t]{2}{*}{ Mean } & \multirow[t]{2}{*}{ SD } & \multirow[t]{2}{*}{$n$} & \multirow[t]{2}{*}{ Mean } & \multirow[t]{2}{*}{ SD } & & & & \\
\hline & & & & & & & & & & \\
\hline \multicolumn{11}{|l|}{ Physical $^{3,5}$} \\
\hline Physical function & 110 & 75.4 & 23.1 & 2886 & 85.7 & 22.1 & 2.4 & -1.8 & 6.6 & 0.25 \\
\hline Role physical & 110 & 63.9 & 40.4 & 2856 & 83.7 & 31.7 & -9.2 & -16.9 & -1.4 & 0.021 \\
\hline Bodily pain & 110 & 69.7 & 27.6 & 2905 & 79.1 & 27.4 & -1.6 & -6.7 & 3.5 & 0.53 \\
\hline General health & 110 & 61.0 & 18.1 & 2859 & 68.1 & 20.2 & 1.0 & -2.5 & 4.6 & 0.57 \\
\hline $\mathrm{PCS}^{4,6}$ & 110 & 46.2 & 10.3 & 2773 & 50.2 & 10.2 & 2.3 & 0.4 & 4.2 & 0.017 \\
\hline \multicolumn{11}{|l|}{ Mental $^{3,5}$} \\
\hline Vitality & 110 & 54.7 & 17.6 & 2876 & 63.3 & 18.5 & -4.8 & -8.2 & -1.4 & 0.006 \\
\hline Social function & 110 & 75.9 & 24.4 & 2911 & 88.8 & 18.4 & -10.2 & -14.9 & -5.6 & $<0.001^{2}$ \\
\hline Role emotional & 110 & 70.6 & 40.6 & 2855 & 90.4 & 25.6 & -16.6 & -24.3 & -8.9 & $<0.001^{2}$ \\
\hline Mental health & 110 & 63.9 & 18.7 & 2871 & 73.9 & 16.4 & -10.2 & -13.7 & -6.7 & $<0.001^{2}$ \\
\hline $\operatorname{MCS}^{4,6}$ & 110 & 45.9 & 11.1 & 2773 & 51.5 & 8.1 & -6.4 & -8.5 & -4.3 & $<0.001^{2}$ \\
\hline AES & 110 & 33.0 & 8.3 & 37 & 23.5 & 5.8 & 9.5 & 6.6 & 12.5 & $<0.001^{2}$ \\
\hline STAI-T $^{5}$ & 110 & 39.8 & 11.3 & 2385 & 35.8 & 9,5 & 3.7 & 1.6 & 5.9 & $0.001^{2}$ \\
\hline BDI & 110 & 8.0 & 6.5 & 86 & 6.5 & 5.2 & 1.5 & -0.2 & 3.2 & 0.081 \\
\hline ESS & 110 & 6.6 & 3.4 & 159 & 5.7 & 3.0 & 0.9 & 0.1 & 1.7 & 0.021 \\
\hline FAB & 110 & 16.2 & 1.8 & 42 & 17.3 & 0.8 & -1.1 & -1.5 & -0.7 & $<0.001^{2}$ \\
\hline PANDA-cognition & 110 & 19.3 & 5.6 & 108 & 23.6 & 5.4 & -4.3 & -5.8 & -2.9 & $<0.001^{2}$ \\
\hline Sniffin Sticks ${ }^{6}$ & 110 & 8.8 & 2.3 & 1012 & 10.2 & 2.1 & -0.3 & -0.7 & 0.1 & 0.139 \\
\hline
\end{tabular}

Abbreviations: AES, Apathy Evaluation Score; BDI, Beck Depression Inventory; ESS, Epworth Sleepiness Scale; ET, Essential Tremor; FAB,

Frontal Assessment Battery; MCS, Mental Component Score; PANDA, Parkinson Neuropsychometric Dementia Assessment; PCS, Physical

Component Score; SD, Standard Deviation; SF-36, Short-Form 36-Item Health Survey; STAI-T, State-Trait Anxiety Scale.

${ }^{1}$ For details of the control subjects refer to the methods and literature.

${ }^{2}$ Remains significant after Bonferroni-Holm adjustment (multiple testing).

${ }^{3}$ Transformed scores.

${ }^{4}$ Normalized scores.

${ }^{5}$ Stratified by age and sex.

${ }^{6}$ Stratified by age.

${ }^{7}$ Compared using Student's t-tests.

bias. ${ }^{17}$ In the present study, patients were recruited using an informative newspaper article in the local media, thereby producing a study population that consisted mainly of patients who had never sought specialist care. The epidemiologic data of our 110 participants with definite and probable ET are in accordance with previous publications regarding motor symptoms, tremor localization, age, alcohol sensitivity, positive family history, AAO, and DD. ${ }^{33}$
The applied test battery identified a pattern of neuropsychiatric findings and QoL changes. We were able to reveal that ET patients experience higher apathy and trait anxiety and exhibit a measurably impaired cognitive function. Regarding QoL measured with the SF36 , we found a statistically significant difference in three out of four MCS subdomains compared to a normal German population; these findings are similar to those reported by Lorenz et al. ${ }^{17}$ Regarding the 


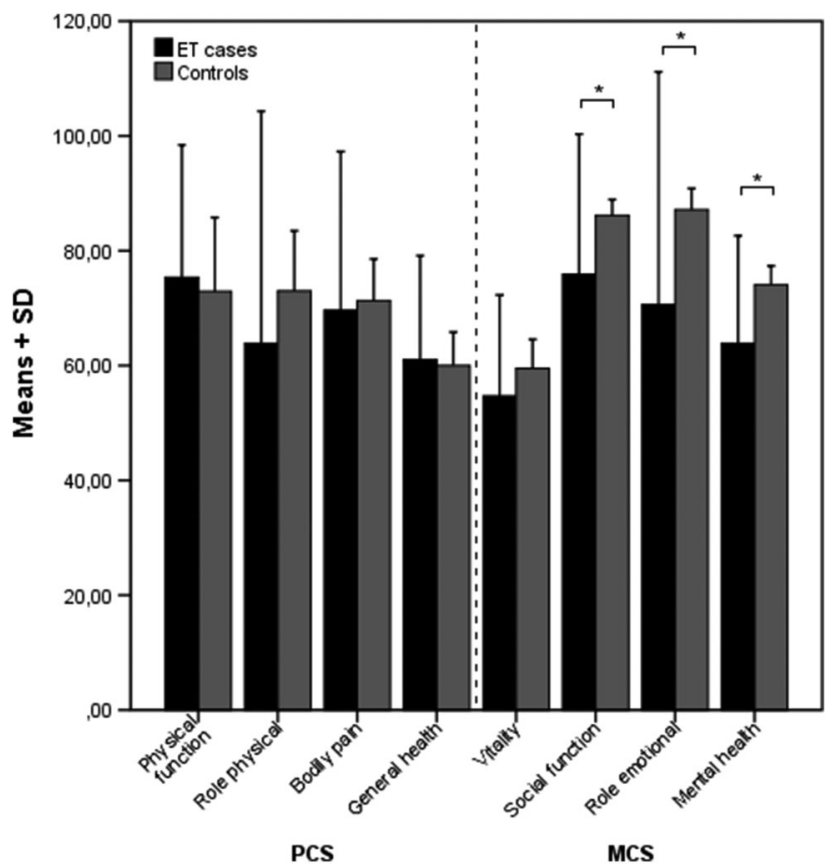

Figure 2. Means and SDs of Patients with ET and Controls on SF-36 Subdomains. Significant after Bonferroni-Holm correction. $* p<0.001$. ET, Essential Tremor; SD, Standard Deviation; SF-36, Short-Form 36-Item Health Survey.

"role emotional" subdomain of the MCS, there was no correlation with tremor severity measured by FTRS and tremor duration. For "mental health" and "social function," we found weak correlations with FTRS but no correlation with tremor duration. This is in contrast to the greater changes in absolute values of all subdomains reported by Lorenz et al. ${ }^{17}$ This could be an effect of the possible selection bias due to the use of outpatients as a study population. Hypothetically, these patients actively seek specialist care because they suffer more from their motor symptoms than the average ET patient. The PCS showed a weak negative correlation with disease severity; however, we did not observe any significant differences in PCS or its subdomains between ET patients and controls. The MCS and PCS findings suggest that reduced QoL in ET patients is mainly caused by mental factors rather than physical impairment and might be classified as intrinsic NMS of ET.

Apathy was the major neuropsychiatric symptom in our sample of ET patients; this symptom is observed in a wide spectrum of psychiatric disorders (e.g., depression or personality disorders) but is also an NMS in movement disorders like PD. ${ }^{34}$ In line with the study by Louis et al., ${ }^{11}$ we observed a highly significant increase in the AES score but could not find a correlation with tremor severity or DD. It could be hypothesized that apathy is even more distinct in ET because patients with severe apathy might not have responded to our recruitment method. Another highly significant aberration was observed in trait anxiety independent of tremor characteristics, especially tremor severity. In the past, anxiety in tremor patients correlated with functional disability. ${ }^{32}$ In two other studies of
Turkish $^{15}$ and Asian ${ }^{35}$ cohorts, anxiety was reported as a concomitant symptom of ET. Interestingly, although apathy and trait anxiety can be clinical symptoms of depression, we did not find a significant change in BDI. This contrasts with the small study by Lacritz et al. ${ }^{13}$ conducted with only 13 ET patients. Studies with larger cohorts ${ }^{15,36}$ reported significant changes in depressive mood scores using scales other than the BDI, which makes comparison difficult. We found a significant correlation of BDI scores with AES, ESS, and STAI-T scores, likely due to the fact that the BDI subitems measure apathy, anxiety, and sleep irregularities. These correlations underscore the quality and reliability of our results. Regarding cognitive impairment, several authors have reported that there are cognitive deficits in ET in several cognitive subdomains. ${ }^{8,12,37,38}$ The available studies differ greatly in the numbers of assessed patients, and the age distribution of the cohort also plays an important role. In our mixed study population, we found a highly significant decline in the FAB test battery; this is similar to former studies measuring frontal executive function in ET patients. ${ }^{38-40}$ However, the mean FAB value in our study was within the normal range of healthy controls, and the significant difference of one point in our sample probably has no clinical impact. To our knowledge, we are the first to use the PANDA-cognition test to assess ET patients. We found a significant decrease with a marginally higher mean value toward the cut-off for mild cognitive dysfunction, and this correlated with tremor severity as measured by FTRS-score. Dementia in PD is considered to be subcortical and thereby also consists of frontal executive dysfunction that can be measured with the PANDA. Because all of our abnormal findings (apathy, anxiety, and frontal

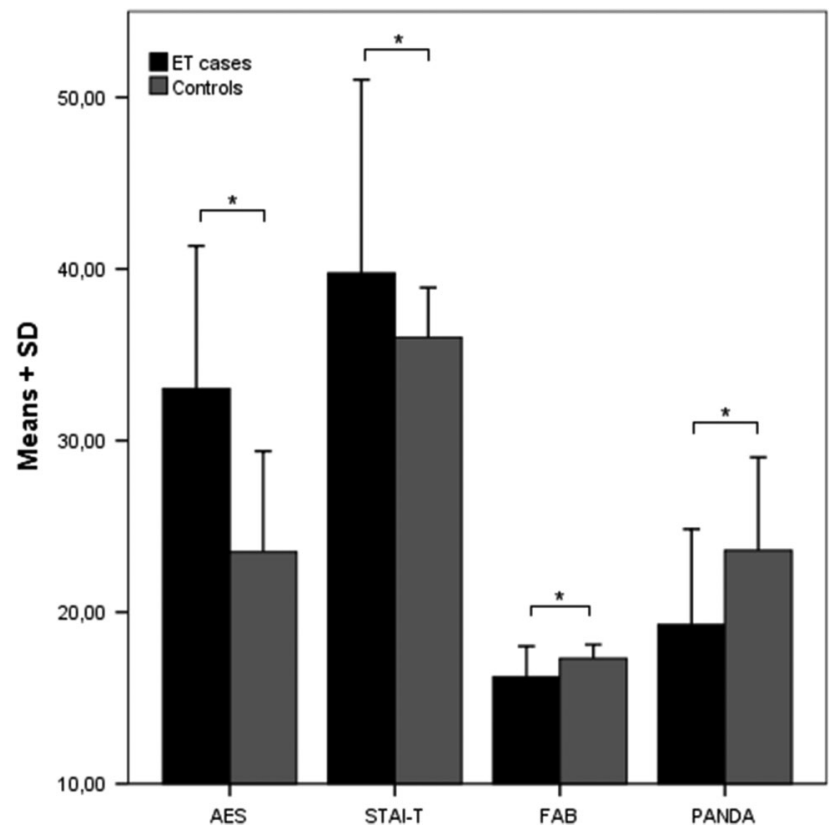

Figure 3. Means and SDs of Patients with ET and Controls on the AES, STAI-T, FAB, and PANDA. Significance after Bonferroni-Holm correction. $* p \leq 0.001$. AES, Apathy Evaluation Scale; ET, Essential Tremor; FAB, Frontal Assessment Battery; PANDA, Parkinson Neuropsychometric Dementia Assessment; SD, Standard Deviation; STAI-T, State-Trait Anxiety Trait. 


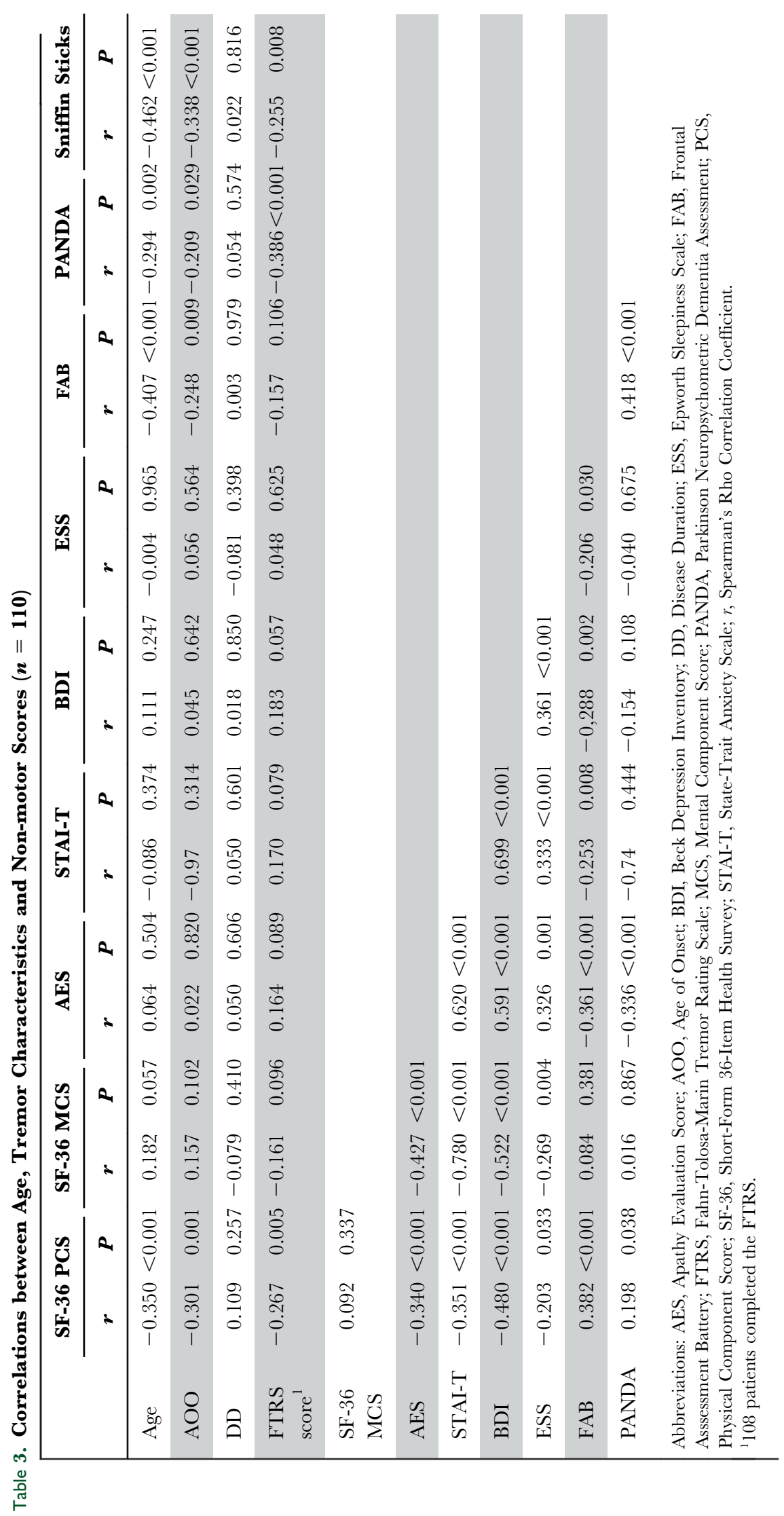


executive function) converge anatomically to frontotemporal and mesolimbic structures, imaging studies should be performed to assess these brain regions in ET.

We did not observe a significant difference in daytime sleepiness between subjects with ET and data from normative controls, which is in accordance with several previous studies that administered the ESS to ET patients. ${ }^{14,39}$ Contrary to previous reports, ${ }^{40}$ olfactory testing did not reveal any significant aberrations suggesting that ET is not characterized by olfactory system neurodegeneration.

This study has some limitations that have to be pointed out, most notably the absence of a control group. Instead, we compared the ET data with standardized psychometric measurements and think this is applicable because there was no intervention and the data were collected at a single time point. Nevertheless, it would be useful to include a control group in subsequent studies to verify our results. Additionally, further longitudinal investigations dealing with NMS in ET should be designed to observe changes over time. Our recruitment method could be limited by selection bias (e.g., only more interested or well-educated patients could have answered the newspaper article), but we consider that it was more appropriate than clinic-based sampling. Another interesting topic to pursue would be subgroup analysis (e.g., sex, distribution of tremor, medication). Our sex-specific subgroup analysis did not reveal a significant difference between male and female participants (data not shown). Therefore, a larger cohort would be more appropriate for future subgroup analyses.

In conclusion, we identified a pattern of NMS in ET using a broad battery of neuropsychiatric and QoL tests in a community setting. The reported epidemiologic and clinical findings of ET symptoms seem to be representative for the known disease phenotype. In our view, the significant neuropsychiatric deficits (apathy, anxiety, frontal executive dysfunction) and reduced QoL of ET patients themselves demonstrate a degree of illness that may be regarded as a non-motor phenotype rather than a secondary effect of motor symptoms. While our results are not clinically obvious, these deficits could be part of a psychiatric premorbid condition. In the future, NMS should be carefully explored in ET patients because they might affect QoL and treatment. Longitudinal studies are required to clarify the non-motor phenotype in ET and learn more about the development and course of the reported abnormalities.

\section{References}

I. Bain P, Brin M, Deuschl G, et al. Criteria for the diagnosis of essential tremor. Neurology 2000;54:S7.

2. Elble RJ. Essential tremor is a monosymptomatic disorder. Mov Disorder 2002;17:633-637, doi: http://dx.doi.org/10.1002/mds. 10222.

3. Benito-León J. Essential tremor: from a monosymptomatic disorder to a more complex entity. Neuroepidemiology 2008;31:191-192.

4. Bonuccelli U. Essential tremor is a neurodegenerative disease. $f$ Neural Transm (Vienna) 2012;119:1383-1387; discussion 73, doi: http://dx.doi.org/ 10.1007/s00702-012-0878-8.

5. Louis ED, Faust PL. Purkinje cell loss in essential tremor. Mov Disorder 2014;29:1329-1330, doi: http://dx.doi.org/10.1002/mds.25965.
6. Louis ED, Vonsattel JP. The emerging neuropathology of essential tremor. Mov Disorder 2008;23:174-1782, doi: http://dx.doi.org/10.1002/mds.21731.

7. Lorenz D, Deuschl G. Update on pathogenesis and treatment of essential tremor. Curr Opin Neurol 2007;20:447-452, doi: http://dx.doi.org/10.1097/ WCO.0b013e3281e66942.

8. Benito-Leon J, Louis ED, Bermejo-Pareja F. Population-based casecontrol study of cognitive function in essential tremor. Neurology 2006;66:69-74, doi: http://dx.doi.org/10.1212/01.wnl.0000192393.05850.ec.

9. Thawani SP, Schupf N, Louis ED. Essential tremor is associated with dementia: prospective population-based study in New York. Neurology 2009;73: 621-625, doi: http://dx.doi.org/10.1212/WNL.0b013e3181b389f1.

10. Bermejo-Pareja F, Puertas-Martín V. Cognitive features of essential tremor: a review of the clinical aspects and possible mechanistic underpinnings. Tremor Other Hyperkinet Mov (N Y) 2012;2, doi: http://dx.doi.org/10.7916/D89W0D7W.

II. Louis ED, Huey ED, Gerbin M, Viner AS. Apathy in essential tremor, dystonia, and Parkinson's disease: a comparison with normal controls. Mov Disorder 2012;27:432-434, doi: http://dx.doi.org/10.1002/mds.24049.

12. Lombardi WJ, Woolston DJ, Roberts JW, Gross RE. Cognitive deficits in patients with essential tremor. Neurology 2001;57:785-790, doi: http://dx.doi. org/10.1212/WNL.57.5.785.

13. Lacritz LH, Dewey R Jr, Giller C, Cullum CM. Cognitive functioning in individuals with "benign" essential tremor. I Int Neuropsychol Soc 2002;8: 125-129, doi: http://dx.doi.org/10.1017/S1355617702001121.

14. Chandran V, Pal PK, Reddy JY, Thennarasu K, Yadav R, Shivashankar N. Non-motor features in essential tremor. Acta Neurol Scand 2012;125:332-337, doi: http://dx.doi.org/10.1111/j.1600-0404.2011.01573.x.

15. Dogu O, Sevim S, Camdeviren H, et al. Prevalence of essential tremor: door-to-door neurologic exams in Mersin Province, Turkey. Neurology 2003;61: 1804-1806, doi: http://dx.doi.org/10.1212/01.WNL.0000099075.19951.8C.

16. Benito-León J, Louis ED, Bermejo-Pareja F. Short sleep duration heralds essential tremor: a prospective, population-based study. Mov Disorder 2013;28: 1700-1707, doi: http://dx.doi.org/10.1002/mds.25590.

17. Lorenz D, Schwieger D, Moises H, Deuschl G. Quality of life and personality in essential tremor patients. Mov Disorder 2006;21:1114-1118, doi: http://dx.doi.org/10.1002/mds.20884.

18. Deuschl G, Bain P, Brin M. Consensus statement of the Movement Disorder Society on Tremor. Ad Hoc Scientific Committee. Mov Disorder 1998;13 Suppl 3:2-23.

19. Bullinger M. German translation and psychometric testing of the SF-36 Health Survey: preliminary results from the IQOLA Project. Int Qual Life Assess Soc Sci Med 1995;41:1359-1366.

20. Bullinger M, Kirchberger I. SF-36 Fragebogen zum Gesundheitszustand. Handanweisung.: Hogrefe, Göttingen, 1998.

21. Marin RS, Biedrzycki RC, Firinciogullari S. Reliability and validity of the Apathy Evaluation Scale. Psychiatr Res 1991;38:143-162, doi: http://dx.doi. org/10.1016/0165-1781(91)90040-V.

22. Lueken U, Seidl U, Schwarz M, et al. [Psychometric properties of a German version of the Apathy Evaluation Scale]. Fortschritte Neurol Psychiatr 2006;74:714-722, doi: http://dx.doi.org/10.1055/s-2006-932164. Die Apathy Evaluation Scale: Erste Ergebnisse zu den psychometrischen Eigenschaften einer deutschsprachigen Ubersetzung der Skala. 
23. Laux VL, Glanzmann P, Schaffner P, et al. STAI State-Trait-Angstinventar.: Beltz, Weinheim, 1981.

24. Hautzinger M, Bailer M, Worall H, et al. Beck-depressions-inventar (BDI). Testhandbuch: Huber, Bern, 1995.

25. Bloch KE, Schoch OD, Zhang JN, Russi EW. German version of the Epworth Sleepiness Scale. Respiration 1999;66:440-447, doi: http://dx.doi.org/ 10.1159/000029408.

26. Dubois B, Slachevsky A, Litvan I, Pillon B. The FAB: a frontal assessment battery at bedside. Neurology 2000;55:1621-1626, doi: http://dx.doi. org/10.1212/WNL.55.11.1621.

27. Kalbe E, Calabrese P, Kohn N, et al. Screening for cognitive deficits in Parkinson's disease with the Parkinson neuropsychometric dementia assessment (PANDA) instrument. Parkinsonism Relat Disord 2008;14:93-101, doi: http://dx.doi.org/ 10.1016/j.parkreldis.2007.06.008.

28. Hummel T, Konnerth CG, Rosenheim K, Kobal G. Screening of olfactory function with a four-minute odor identification test: reliability, normative data, and investigations in patients with olfactory loss. Ann Otol Rhinol Laryngol 2001;110:976-981, doi: http://dx.doi.org/10.1177/000348940111 001015 .

29. Lageman SK, Cash TV, Mickens MN. Patient-reported needs, non-motor symptoms, and quality of life in essential tremor and Parkinson's disease. Tremor Other Hyperkinet Mov (N Y) 2014;4, doi: http://dx.doi.org/10.7916/D8RF5S4J.

30. Gasparini M, Bonifati V, Fabrizio E, et al. Frontal lobe dysfunction in essential tremor: a preliminary study. $\mathcal{J}$ Neurol 2001;248:399-402, doi: http:// dx.doi.org/10.1007/s004150170181.

31. Jhunjhunwala K, Pal PK. The non-motor features of essential tremor: a primary disease feature or just a secondary phenomenon? Tremor Other Hyperkinet $\operatorname{Mov}(\mathcal{N}$ r) 2014;4, doi: http://dx.doi.org/10.7916/D8D798MZ.
32. Louis ED, Barnes L, Albert SM, et al. Correlates of functional disability in essential tremor. Mov Disorder 2001;16:914-920.

33. Romero JP, Benito-León J, Bermejo-Pareja F. The NEDICES study: recent advances in the understanding of the epidemiology of essential tremor. Tremor Other Hyperkinet Mov (N V) 2012;2, doi: http://dx.doi.org/10.7916/D8N58K4H.

34. Kirsch-Darrow L, Fernandez HH, Marsiske M, Okun MS, Bowers D. Dissociating apathy and depression in Parkinson disease. Neurology 2006;67:3338, doi: http://dx.doi.org/10.1212/01.wnl.0000230572.07791.22.

35. Tan EK, Fook-Chong S, Lum SY, et al. Non-motor manifestations in essential tremor: use of a validated instrument to evaluate a wide spectrum of symptoms. Parkinsonism Relat Disord 2005;11:375-380, doi: http://dx.doi.org/ 10.1016/j.parkreldis.2005.04.007.

36. Louis ED, Benito-León J, Bermejo-Pareja F; Neurological Disorders in Central Spain (NEDICES) Study Group. Philadelphia Geriatric Morale Scale in essential tremor: a population-based study in three Spanish communities. Mov Disorder 2008;23:1435-1440, doi: http://dx.doi.org/10.1002/mds.22124.

37. Sahin HA, Terzi M, Uçak S, Yapici O, Basoglu T, Onar M. Frontal functions in young patients with essential tremor: a case comparison study. I Neuropsychiatr Clin Neurosci 2006;18:64-72, doi: http://dx.doi.org/10.1176/ jnp. 18.1.64.

38. Park IS, Oh YS, Lee KS, et al. Subtype of mild cognitive impairment in elderly patients with essential tremor. Alzheimer Dis Assoc Disorder 2015;29:141-145.

39. Gerbin M, Viner AS, Louis ED. Sleep in essential tremor: a comparison with normal controls and Parkinson's disease patients. Parkinsonism Relat Disord 2012;18:279-284, doi: http://dx.doi.org/10.1016/j.parkreldis.2011.11.004.

40. Louis ED, Bromley SM, Jurewicz EC, Watner D. Olfactory dysfunction in essential tremor: a deficit unrelated to disease duration or severity. Neurology 2002;59:1631-1633, doi: http://dx.doi.org/10.1212/01.WNL.0000033798. 85208.F2. 